Ann. Zootech., I975, 24 (3), 565-570.

NOTE

\title{
COMPOSITION CHIMIQUE ET VALEUR ALIMENTAIRE D'UNE MAUVE (MALVA VERTICILLATA L.) (PREMIERS RÉSULTATS)
}

\author{
J. BONNEMAIRE, M. ROUX et J. H. TEISSIER \\ avec la collaboration technique de Catherine CoRdiLET et L. GRENET \\ Laboratoive de Recherches de la Chaive de Zootechnie, \\ Centre do Recherches de Dijon, I. N. R. A., \\ Ecolo nationale supérieure des Sciences agronomiques appliquées,
} 21016 Dijon Cedex

\begin{abstract}
RÉSUMÉ
L'évolution de la digestibilité et de la quantité ingérée de la plante entière de mauve (Malva verticillata L.) a été mesurée sur des moutons au cours des $\mathrm{I}^{\mathrm{er}}$ et $2^{\mathrm{e}}$ cycles de végétation.

$\Lambda u$ cours du I er cycle ( 5 semaines), la teneur en matière sèche a augmenté de 20 ì $28 \mathrm{p}$. Ioo; la teneur en cellulose brute a augmenté de I 9 à $28 \mathrm{p}$. Ioo tandis que le taux de matières minérales et le taux de matières azotées ont diminué respectivement de I4 à $10 \mathrm{p}$. Ioo et de 20 à I 3 p. Ioo, environ. La digestibilité de la matière organique a diminué de 72 à 66 p. Ioo environ. Les quantités ingérées se sont situécs à un niveau élevé : 103,2 à $95,7 \mathrm{~g}$ de $\mathrm{MS} / \mathrm{kg} \mathrm{P}^{0,75}$.

Les repousses âgées de 7 semaines contenaient environ 13 p. Ioo de matière sèche, I 8 à 24 p. roo de cellulose brute, I 7 à $24 \mathrm{p}$. roo de matières minérales, I9 à 24 p. Ioo de matières azotées ; la digestibilité de la matière organique et les quantités ingérées sont restées comprises respectivement entre 75 et $80 \mathrm{p}$. Ioo et 88 et $96 \mathrm{~g}$ de MS $/ \mathrm{kg} \mathrm{P}^{0.75}$.

I.a production maximum de matière sèche récoltée à l'hectare a été pour les deux coupes d'environ 7.5 tonnes.
\end{abstract}

\section{INTRODUCTION}

La plante de mauve est utilisée comme fourrage dans un certain nombre de régions d'Asie et d'Europe de l'Est, en particulier dans des zones de conditions écologiques difficiles. Ainsi avonsnous observé dans une vallée de l'Himalaya Central (3 70o $\mathrm{m}$ d'altitude) qu'elle atteint un développement végétatif important et qu'elle y jouc, une fois séchéc, un rôle essentiel dans l'alimen- 
tation hivernale des bovins et des yaks (Bonnemaire et Terssier, I973). Il s'agit dans ce cas de l'espèce_Malva verticillata connue depuis longtemps cn Chine et étudiée d'un point de vue botanique pat DANERT (1965, 1966).

De nombreux travaux ont été réalisés en U.R.S.S. sur différentes espèces fourragères de mauve, et en particulier : Malva meluca, Malva crispa ct Malva verticillata. SoKolov et Yakimov (1967) soulignent leur intérêt comme plante à ensiler en raison de leur résistance au froid, de leur précocité ct des bons rendements en fourrage vert ; Malva meluca serait plus productive que Malva verticillata et Malva crispa, quoique d'un développement moins rapide. Ces études russes ont été conduites dans différentes régions de ce pays, essentiellement dans la partie septentrionale, depuis la Lithuanie jusqu'ì la Sibérie Orientale (province de Yakut) -- -, où les conditions naturelles limitent de manière importante les potentialités de nombrcuses plantes fourragères. Les essais relatés font état de résultats de production très divers (de 20 à $87 \mathrm{t}$ de fourrage vert/ha) ; ils fournissent des indications sur les conditions de production, la composition chimique et la valeur alimentaire de ces plantes dans divers cas d'utilisation : vert ou ensilage, mauve seule ou mauve en mélange avec d'autres plantes (en particulier en semis alterné avec du maïs). Ainsi, en 1970, s'est tenu à Leningrad un symposium sur de nouvelles plantes à ensiler (SoкоLov, 1970) où la mauve apparaît dans 22 communications sur 162 ; il en ressort notamment que cette plante assez pauvre en glucides solubles est souvent ensilée avec addition d'un conservateur ou de mélasse, ou bien en mélange avec du maïs ou une autre plante. Une étude de Malva crispa conservée sous forme d'ensilage a également été entreprise en Belgirue (CoTTYN et al., 1970).

Les données de la bibliographie viennent donc appuyer les observations que nous avions pu faire en altitude sur la capacité de production de cette plante et son rôle dans l'alimentation animale. On peut alors s'interroger sur l'intérêt éventuel, - fut-il très marginal —, que de telles plantes pourraient être amenées à présenter dans certains secteurs défavorisés. C'est pourquoi, dans le cadre des études conduites au Laboratoire de la Chaire de Zootechnie de l'E. N. S. S. A. A. sur la valeur alimentaire des fourrages, nous avons entrepris en 1974 une étude sur Malva verticillata. Ce travail a été réalisé à partir d'un échantillon de graines collectées au Népal en 1972, et multipliées au domaine expérimental de Dijon-Eepoisses par PICARD en 1973. Cette première année d'expérimentation nous a permis d'enregistrer des indications sur le rendement de cette mauve et d'étudier l'évolution de sa composition chimique et morphologique, de sa valeur nutritive et de son ingestibilité par des moutons maintenus en cage à métabolisme.

\section{MATÉRIEL E'T MÉTHODES}

La digestibilité et la quantité ingérée de la plante entière de mauve ont été mesurées sur des moutons pendant 5 semaines consécutives, du 28 juillet au 29 août, pour le I er cycle et pendant 4 semaines consécutives, du I $_{5}$ septembre au i 1 actobre, pour le $2^{\mathrm{e}}$ cycle.

La mauve a été semée le 9 mai 1974 dans une parcelle du domaine de la Station d'Amélioration des Plantes d'Epoisses en Côte-d'Or. Afin d'améliorer le taux de germination, - très faible naturellement (observations de PICARD (1973), confirmées par divers auteurs et notamment SoKolov et YAKIMOV, I 967) -, nous avons, avant le semis, immergé les graines pendant Io minutes dans l'azote liquide. Le semis a été effectué en ligne avec un écartement de 50 centimètres entre les lignes et une densité d'environ $7^{\circ}$ plantes au mètre carré. La levée a eu lieu le I 8 mai 1974. Deux binages ont été nécessaires dans le courant du mois de juin. La fertilisation reçue a été de $\mathbf{I} 20$ unités de $\mathrm{P}$ et $\mathrm{K}$ à l'hectare en fumure d'hiver et de 80 unités d'azote à l'hectare le 2 juillet. D'autre part la parcelle fut irriguée respectivement les ${ }_{1}$ er et 29 aout par 60 et $50 \mathrm{~mm}$ d'eau.

La mauve était fauchée 3 fois par semaine, les lundi, mercredi ct vendredi ; la quantité récoltée était pesée et la surface fauchée mesurée chaque fois. La récolte du lundi servait à alimenter les moutons les mardi et mercredi, celle du mercredi à les alimenter les jeudi et vendredi, et celle du vendredi à les alimenter du samedi au lundi. Dès la récolte la mauve était hachée dans un hache-paille en brins de 2 à $3 \mathrm{~cm}$ de longueur et stockée en chambre froide $\left(+4^{\circ} \mathrm{C}\right)$ jusqu'à la 
distribution aux animaux. Celle-ci a été distribuée à volonté (5 à Io pour roo de refus) chaque jour en 2 repas $(9 \mathrm{~h}$ et $\mathrm{I} 7 \mathrm{~h}$ à un lot de 4 moutons en cage à métabolisme. La digestibilité et la quantité ingérée ont été mesurées en continu; chaque période de mesure allant du dimanche au samedi. La composition morphologique de la plante a été déterminée pendant 7 semaines consécutives pour le I er cycle et 3 fois au cours du $2^{\mathrm{e}}$ cycle (à I 5 jours d'intervalle). A cet effet, un échantillon de 20 plantes a été prélevé ; pour chaque plante, nous avons, après avoir mesuré sa hauteur, séparé les feuilles des tiges. Ces deux fractions ont été séchées à l'étuve puis pesées et leur poids ont été exprimés en p. Ioo du poids de la matière sèche de la plante.

Les échantillons représentatifs de la mauve distribuéc, de la mauve refusée et des fèces correspondant à chaque période ont été analysés pour déterminer leurs teneurs en cendres, matières azotées et en cellulose brute de Weende.

\section{RÉSULTATS EIT DISCUSSION}

\section{1. - Composition chimique et morphologique}

La participation relative des divers organes à la matière sèche de la plante a évolué régulièrement au cours du premier cycle. La part des tiges a augmenté de 55 à 80 p. Ioo au détriment de celle des feuilles qui est passée de 45 à 20 p. ıoo. En fin cle cycle, les graines n'ont représenté que 5 à $6 \mathrm{p}$. Ioo de la matière sèche. Au deuxième cycle, la part des tiges a peu évolué et est restée comprise entre 62 et $7 \mathrm{x}$ p. Ioo, mais il est vrai que les repousses étudiées avaient toutes le même âge ( 48 ou 49 jours); en effet, les faibles superficies disponibles n'ont pas permis de suivre l'évolution du deuxième cycle en fonction de l'âge des repousses.

La teneur en matière sèche de la plante a augmenté de 20 à $27 \mathrm{p}$. 100 au premier cycle (tabl. 1). L'augmentation est assez faible compte tenu de la proportion très importante de tiges à la fin des mesures. Cependant, ces teneurs sont nettement plus élevées que celles observées par d'autres auteurs, notamment Laguta (1963) et CotTyn et al. (1970). Au deuxième cycle, elle a été de $\mathbf{1 3}$ p. roo environ, ce qui est une valeur plus proche des données de la bibliographie. La teneur en cendres de la plante de mauve est élevée; clle a diminué de I 4 à Io-I I p. Ioo au cours des cinq scmaines d'étude du premier cycle; pour les repousses du deuxième cycle, elle est restée comprise entre I 7 et 24 p. Ioo. Le fait que, par suite du temps très pluvieux, le fourrage ait été quelque peu souillé par de la terre explique probablement en partie des teneurs en cendres aussi élevées au deuxième cycle. De même, la teneur en matières azotées est relativement élevée : elle a diminué au premier cycle avec le stade de maturité, de is à 13 p. Ioo, et s'est située au deuxième cycle entre 19 et $24 \mathrm{p}$. Ioo. Quant à la teneur en cellulose brute, elle a augmenté au premier cycle de r9 à 28 p. Ioo, et au deuxième cycle elle a varié dans l'intervalle i $8-24$ p. Ioo. Ces résultats sont assez semblables à ceux que l'on trouve dans la bibliographie pour les différentes variétés de mauve (Laguta, 1963; Kruglov, 1965; Egorova et al., 1965; Sukhov, 1966; Sokolov et Yakimov, i967; Cottrn et al., 1970).

\section{2. - Digestibilité}

Pendant les trois premières semaines de mesure du premier cycle, la digestibilité de la matière organique est restée pratiquement constante (de l'ordre de 7 I $^{-72}$ p. Ioo) ; elle a ensuite diminué rapidement de 5 points pour se stabiliser en $4^{\mathrm{e}}$ et $5^{\mathrm{e}}$ semainc aux alentours de $66 \mathrm{p}$. roo (tabl. $\mathrm{I}$ ). L'évolution de la digestibilité de la plante avec l'âge semble donc assez différente de celle observée avec la plupart de nos plantes fourragères. La digestibilité de la cellulose brute a augmenté régu-

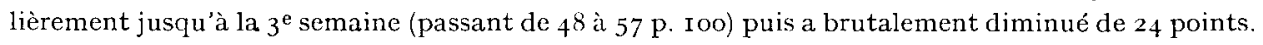
Cette diminution n'a pas été accompagnée d'une augmentation de la teneur en cellulose brute de la plante, comme on l'observe généralement. C'est probablement la très forte lignification des tiges, que l'on a pu observer à partir du 20 août qui peut expliquer cette chute de la digestibilité. La digestibilité des matières azotées a varié à peu près dans lo même sens que la teneur en matières azotées de la plante. 


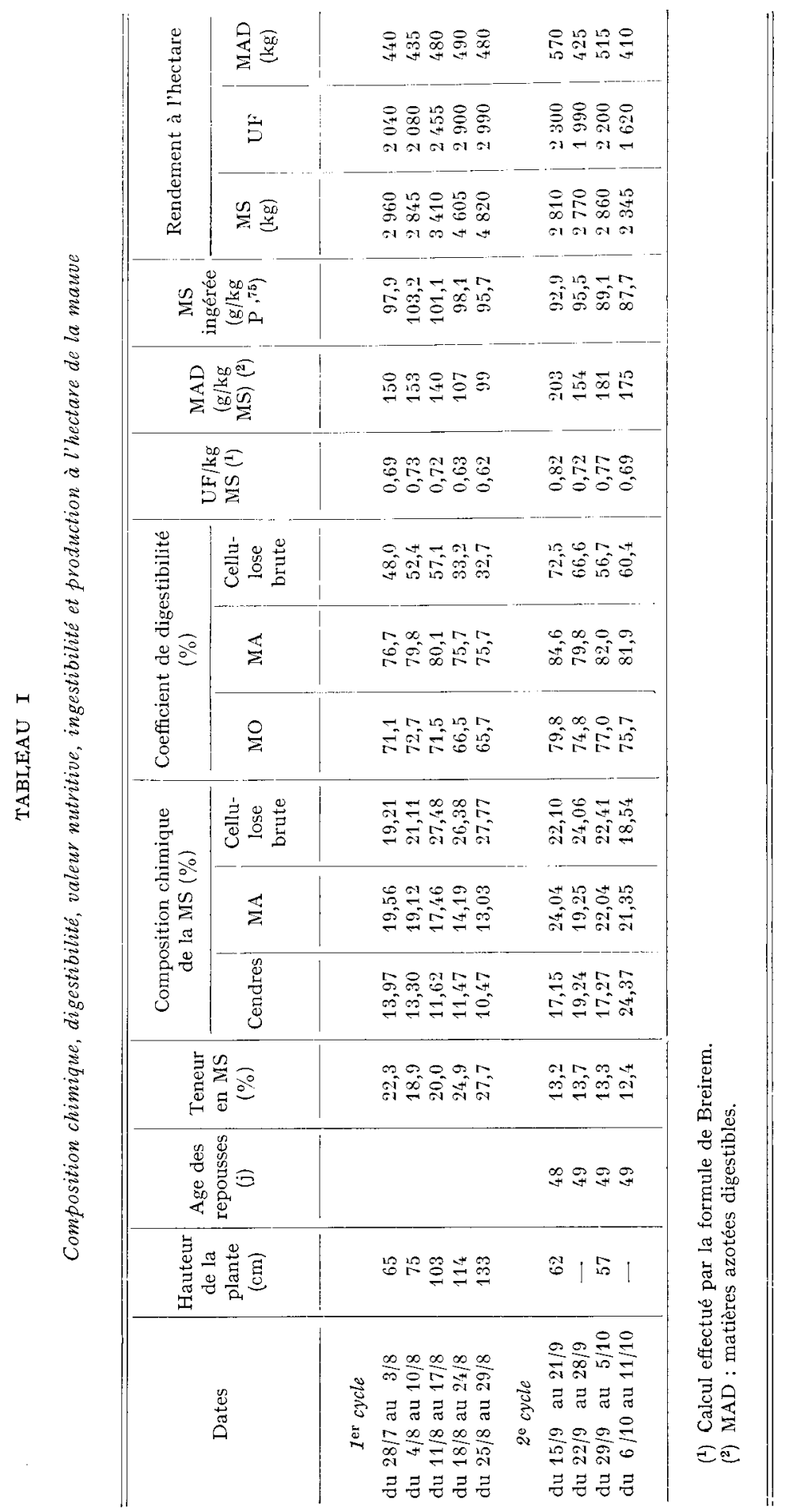


Au deuxième cycle, la digestibilité de la matière organique a été en moyenne plus élevée que pour le premier cycle (variations de 74,8 à 79,8 p. roo) ; la digestibilité de la cellulose brute a eu une évolution relativement indépendante de la teneur en cellulose brute des plantes; la digestibilité des matières azotées a varié parallèlement à la teneur en matières azotées de la plante.

\section{3. - Quantité ingérée}

Au cours du premier cycle, la quantité de matière sèche ingérée par les moutons a été très élevée et relativement constante pendant les cinq semaines de mesure avec cependant une légère diminution de la $2^{\text {è }}$ la $5^{\text {e }}$ semaine (103,2 à 95,7 $\left.\mathrm{g} / \mathrm{kg} \quad \mathrm{P}^{0,75}\right)$.

Le niveau d'ingestion des repousses du deuxième cycle a été en moyenne légèrement plus faible, variant de $87,7 \grave{a ̀} 95,5 \mathrm{~g} / \mathrm{kg} \mathrm{P}^{0,75}$ pendant les quatre semaines de mesure.

\section{4. - Production à l'hectare}

La quantité de matière sèche récoltée à l'hectare au cours du premier cycle a augmenté pendant la période de mesure, mais est restée assez faible; le maximum a été atteint en $5^{\text {e }}$ semaine et s'est situé à $4,8 \mathrm{t}$ de matière sèche. Quant aux quantités d'unités fourragères et de matières azotées digestibles récoltées à l'hectare elles ont atteint leur maximum respectivement durant la $5^{\mathrm{e}}$ et la $4^{\mathrm{e}}$ semaine (2 $990 \mathrm{UF}$ et $490 \mathrm{~kg}$ de MAD).

Compte tenu de l'âge relativement constant des repousses, la quantité de matière sèche récoltée à l'hectare a peu varié pendant les quatre semaines de mesure (en moyenne 2,7 t). Les quantités correspondantes d'unités fourragères et de matières azotées digestibles récoltées à l'hectare ont été en moyenne respectivement de 2 ooo UF et de $480 \mathrm{~kg}$ de MAD.

Globalement, la production maximum de matière sèche récoltée à l'hectare a été pour les deux coupes d'environ 7,5 tonnes. Ce rendement est un peu supérieur à celui enregistré en Belgique (également sur deux coupes) par Cotтy et al. (1970) sur malva crispa (5,9 t de Ms/ha). Toutefois, ces auteurs précisent qu'il s'agit là, pour des raisons particulières, de rendements faibles, et ils indiquent qu'ils pratiquent en général trois coupes (ils annoncent dans ces conditions des rendements voisins de ro $t$ de MS/ha). Quant aux auteurs russes, ils obtiennent des rendements variant de 20 à 87 tonne: de matière verte à l'hectare avec différentes variétés de mauve semées dans des sols ayant reçu des doses d'engrais variables et exploitées différemment, dans des régions aux climats très contrastés (Kruglov, 1965; Medvedev, I965; VarLamova, 1966 ; Marchy ulenis, 1968). Vraisemblablement, dans notre essai, le semis a été trop tardif et la densité insuffisante pour permettre un rendement maximum. Il est probable que des améliorations sur ces points rendraient possible trois coupes et provoqueraient des augmentations notables des rendements.

\section{CONCLUSION}

Cette première année d'étude sur la plante verte de mauve (Malva verticillata L.) a permis de montrer que sa digestibilité est comparable à celle de nombreuses plantes fourragères; son ingestibilité par des moutons s'est révélée, en revanche, très élevée. C'est une plante riche en matières minérales et possédant une bonne teneur en matières azotées. Mais les rendements que nous avons enregistrés demeurent encore assez faibles. Par ailleurs, la chute des graines au fur et à mesure de leur maturation en rend la récolte difficile. Cependant l'ensemble de ces observations demanderait à être confirmé dans d'autres expérimentations. De plus, il conviendrait d'envisager l'utilisation de cette plante sous forme d'ensilage. In fin, il serait intéressant de l'étudier dans des milieux écologiques différents et en particulier dans des zones d'altitude. 


\title{
SUMMARY
}

\author{
CHEMICAL COMPOSITION AND FEEDING VALUE OF MALLOW \\ (MALVA VERTICILLATA I.) (FIRST RESUI,TS)
}

Variation in digestibility and total intake of whole mallow plants (Malva verticillata L.) during the ist and 2 nd growth cycles were measured on sheep.

During the ist cycle (5 weeks) the dry matter content increased from 20 to 28 p. Ioo, that of crude fibre from I 9 to 28 p. Ioo, whereas the levels of minerals and crude protein decreased from about 14 to 10 p. Ioo and from 20 to 13 p. Ioo, respectively. Digestibility of organic matter decreased from about 72 to $66 \mathrm{p}$. Ioo. The feed intake was high : 103.2 to $95.7 \mathrm{~g} \mathrm{DM} / \mathrm{kg} \mathrm{P}^{\mathbf{0 . 7 5}}$.

7 -weeks-old regrowths contained about $13 \mathrm{p}$. Ioo dry matter, i 8 to $24 \mathrm{p}$. Ioo crude fibre, I 7 to $24 \mathrm{p}$. Ioo minerals, I9 to $24 \mathrm{p}$. Ioo crude protein; digestibility of organic matter and the intake levels ranged between 7.5 and $80 \mathrm{p}$. 100, 88 and $96 \mathrm{~g} \mathrm{DM} / \mathrm{kg} \mathrm{P}^{0.75}$, respectively. cuttings.

Maximum amount of dry matter harvested per hectare ranged about 7.5 tons for the two

\section{REMERCIEMINTS}

Nous tenons à remercier tout particulièrement M. J. PICARD, directeur de la Station I. N. R. A. d'Amélioration des Plantes de Dijon qui a bien voulu assurer la multiplication"et la mise en culture de cette plante; son aide et ses suggestions nous ont été très précieuses.

\section{RÉFÉRENCES BIBLIOGRAPHIQUES}

Bonnemaire J., Teissifr J. H., i973. Rapport sur une mission de recherche effectuée au Népal en septembre et octobre 1972. Polycop. $24 \mathrm{p}$.

Cottyn B. G., Boucoue Ch. V., VAN HeE L. P., 197o. Conservation et valeur alimentaire de Malva crispa I. Revue de l'A gric. Bruxelles, 11-12, I667-I68

Danert S., I965. Uber einige infraspezifische Sippen von Malva verticillata I. Kulturpflanze, 13, 715735 .

Dantrt S., 1966. \%ur morphologie und systematik von Malva verticillata L. Kullurpflanze, 14, 495-510.

Egorova A. A., Makeeva I. P., Kunavina V. P., Filippova Z. M., ig65. Biochemical characteristics of fodder crops new to Karelia, in (New fodder-silage plants. Proc and All-Union seminar on new silage plants, Minsk, 8-II. June I964. Minsk, I965, p. 8I-85) (russe) ; (Herb. Abstr., 37, no 2052).

Kíuglov T. L., 1965. Preliminary results of evaluation of malva cultivars promising for the Sverdlovsk Province. Trudy ural's nauchno-issled. Inst. Sel'. Khoz., 6, 198-204 (russe) ; (Herb. Abstr., 37 (I), $n^{\circ}$ I5 I).

Iaguta A. F., I963. Nallow a highly productive fodder crop. Zivosnovodstoo, 10, i2-I5 (russe); (Herb. Abstr., 34 (3), no I 2 I6).

Marchyulenis V. I., I968. Effect of fertilizers on the growth, development and chemical composition of Malva meluca. Trudy Akad. Nauk. Litov. S S R. (Ser. B), $1^{\circ} 2$ (46), 47-6o (russe); (Herb. Abstr., $\left.39, n^{\circ}{ }_{3} 84\right)$.

MedvedeV P. F., I965. A brief summary of work on the introduction into cultivation of fodder species of malva in the USSR. Rastit. Resurs $\bar{y}$, 1965, I, n⿳ $\mathbf{1}$, Io9-1 5 (Sev. - Zap. N. I. Sel'. Khoz. Inst., Leningrad) (russe); (Herb. A bstr., 36, n⿳0 1776).

Picard J., I973. (Communication personnelle.)

Sokolov V. S., Yakimov A. P., I g67. New silage plants in the USSR. Herb. Abstr., 37, $247-250$.

Sokolov V.S. (editor), I97o. Fifth symposium on new silage plants. Parts I and 2. Leningrad, USSR, Botanicheskil Institut (I970) I 22 p. and I I r p.; (russe) ; (Herb. Abstr, 42, no 3463 et suiv.).

Sukrov V. A., I966. The introduction of fodder species of malva to central Yakutia, in (New silage plants. Proc. 3rd symposium on new silage plants, Sȳktȳvkar 9-13 Aug. 1965; Sȳktȳvkar, 1966, p. 70-78) (russe) ; (Herb. A bstr., 37, 4, no 2054).

Varlamova K. A., I966. The effect of photoperiod on mallow sown on different dates. Sel'.-khoz. Biologiya, 1, no 3 , 405-9 (russe); (Herb. Abstr., 37, no 848 ). 